\title{
LINEAR INTEGRAL EQUATIONS OF FUNCTIONS OF TWO VARIABLES*
}

BY T. S. PETERSON

1. Introduction. It is the purpose of this paper to consider certain conditions for the solution of the following linear integral equation:

$$
\begin{gathered}
\bar{y}(\alpha, \beta)=y(\alpha, \beta)+\lambda \int_{a}^{b} K(\alpha, \sigma) y(\sigma, \beta) d \sigma+\mu \int_{a}^{b} L(\beta, \tau) y(\alpha, \tau) d \tau \\
+\nu \int_{a}^{b} \int_{a}^{b} M(\alpha, \beta, \sigma, \tau) y(\sigma, \tau) d \sigma d \tau
\end{gathered}
$$

and, particularly, the truncated form with $M(\alpha, \beta, \sigma, \tau) \equiv 0$. The more important results of the paper are to be found summarized in Theorems 2 and 3.

Throughout the paper we shall consider all given functions as bounded and continuous, and in order to facilitate the work we shall adhere to the notation (1) to represent the variables of functions as indices, (2) to signify by the repetition of an index in a term, once as a subscript and once as a superscript, an integration on that variable over the fundamental interval $(a, b)$.

2. A Generalization of the Fredholm Equation. Let us consider a special type of integral equation of a function of two variables which has as its origin the succession of two ordinary Fredholm equations, namely

$$
\bar{y}^{\alpha \beta}=y^{\alpha \beta}+\lambda K_{v}^{\alpha} y^{\sigma \beta}+\mu L_{\tau}^{\beta} y^{\alpha \tau}+\lambda \mu K_{\sigma}^{\alpha} L_{\tau}^{\beta} y^{\sigma \tau} .
$$

In fact, (1) is given by the succession of equations

$$
z^{\alpha \beta}=y^{\alpha \beta}+\lambda K_{\sigma}^{\alpha} y^{\sigma \beta}, \quad \bar{y}^{\alpha \beta}=z^{\alpha \beta}+\mu L_{\tau}^{\beta} z^{\alpha \tau} .
$$

The equations (2) being ordinary Fredholm equations, it is evident at once that the equation (1) has the unique, continuous inverse

$$
y^{\alpha \beta}=\bar{y}^{\alpha \beta}+\lambda k_{\sigma}^{\alpha} \bar{y}^{\sigma \beta}+\mu l_{\tau}^{\beta} \bar{y}^{\alpha \tau}+\lambda \mu k_{\sigma}^{\alpha} l_{r}^{\beta} \bar{y}^{\sigma \tau},
$$

providing that $\lambda$ and $\mu$ are not characteristic values of their re-

* Presented to the Society, December 29, 1932. 
spective kernels. In equation (3) the small lettered functions represent the respective inverses to the large lettered functions, that is,

$$
k_{\beta}^{\alpha}=-\frac{D_{\beta}^{\alpha}\left[\lambda K_{\tau}{ }^{\sigma}\right]}{D\left[\lambda K_{\tau}{ }^{\sigma}\right]}
$$

where $D_{\beta}{ }^{\alpha}\left[\lambda K_{\tau}{ }^{\sigma}\right]$ and $D\left[\lambda K_{\tau}{ }^{\sigma}\right]$ are the first Fredholm minor and the Fredholm determinant respectively. If either parameter has a characteristic value, say $\lambda=\bar{\lambda}$, then it is evident again that the homogeneous equation (1), that is, $\bar{y}^{\alpha \beta} \equiv 0$, has a solution. Moreover these solutions will be of the form $\Phi_{i}^{\alpha} \phi^{\beta}$, where $\phi^{\beta}$ is an arbitrary function and the $\Phi_{i}{ }^{\alpha},(i=1,2, \cdots, n)$, are the fundamental solutions of the equation

$$
0=f^{\alpha}+\bar{\lambda} K_{\sigma}^{\alpha} f^{\sigma} .
$$

Further properties of equation (1) may be written down at once following the classical theory of Fredholm.

A general equation in which we are more interested, however, is of the following form:

$$
\bar{y}^{\alpha \beta}=y^{\alpha \beta}+\lambda K_{\sigma}^{\alpha} y^{\sigma \beta}+\mu L_{\tau}^{\beta} y^{\alpha \tau}+\nu M_{\sigma \tau}^{\alpha \beta} y^{\sigma \tau} .
$$

To solve equation (4), let us consider the succession of transformations

(5) $z^{\alpha \beta}=y^{\alpha \beta}+\lambda K_{\sigma}^{\alpha} y^{\sigma \beta}, \bar{z}^{\alpha \beta}=z^{\alpha \beta}+\mu L_{\tau}^{\beta} z^{\alpha \tau}, \bar{y}^{\alpha \beta}=\bar{z}^{\alpha \beta}+N_{\sigma \tau}^{\alpha \beta} \bar{z}^{\sigma \tau}$.

These on substitution yield

$$
\begin{aligned}
\bar{y}^{\alpha \beta}=y^{\alpha \beta}+\lambda K_{\sigma}^{\alpha} y^{\sigma \beta} & +\mu L_{\tau}^{\beta} y^{\alpha \tau}+\left\{N_{\sigma \tau}^{\alpha \beta}+\lambda N_{\xi \tau}^{\alpha \beta} K_{\sigma}^{\xi}\right. \\
& \left.+\mu N_{\sigma \eta}^{\alpha \beta} L_{\tau}^{\eta}+\lambda \mu N_{\xi \eta}^{\alpha \beta} K_{\sigma}^{\xi} L_{\tau}^{\eta}+\lambda \mu K_{\sigma}^{\alpha} L_{\tau}^{\beta}\right\} y^{\sigma \tau}
\end{aligned}
$$

which, subject to the conditions that $\lambda, \mu$, and 1 are not characteristic values of the kernels of (5), has the unique, continuous inverse

$$
\begin{aligned}
y^{\alpha \beta}=\bar{y}^{\alpha \beta}+\lambda k_{\sigma}^{\alpha} \bar{y}^{\sigma \beta}+\mu l_{\tau}^{\beta} \bar{y}^{\alpha \tau}+\left\{n_{\sigma \tau}^{\alpha \beta}+\lambda k_{\xi}^{\alpha} n_{\sigma \tau}^{\xi \beta}\right. \\
\\
\left.+\mu l_{\eta}^{\beta} n_{\sigma \tau}^{\alpha \eta}+\lambda \mu k_{\xi}^{\alpha} l_{\eta}^{\beta} n_{\sigma \tau}^{\eta \xi}+\lambda \mu k_{\sigma \tau}^{\alpha} l_{\tau}^{\beta}\right\} \bar{y}^{\sigma \tau}
\end{aligned}
$$

A necessary and sufficient condition that the equation (4) be representable in the form (6) is that we may solve the integral equation

(8) $\nu M_{\sigma \tau}^{\alpha \beta}=N_{\sigma \tau}^{\alpha \beta}+\lambda N_{\xi \tau}^{\alpha \beta} K_{\sigma}^{\xi}+\mu N_{\sigma \tau}^{\alpha \beta} L_{\tau}^{\eta}+\lambda \mu N_{\xi \eta}^{\alpha \beta} K_{\sigma}^{\xi} L_{\tau}^{\eta}+\lambda \mu K_{\sigma}^{\alpha} L_{\tau}^{\beta}$ 
for $N_{\sigma \tau}^{\alpha \beta}$. For $\lambda$ and $\mu$ non-characteristic values this equation may be readily solved as has been noted in the first part of the paper. Doing this, we have

(9) $N_{\alpha \beta}^{\alpha \beta}=\nu M_{\sigma \tau}^{\alpha \beta}+\lambda \nu M_{\xi \tau}^{\alpha \beta} k_{\sigma}^{\xi}+\mu \nu M_{\sigma \eta}^{\alpha \beta} l_{\tau}^{\eta}+\lambda \mu \nu M_{\xi \eta}^{\alpha \beta} k_{\sigma}^{\xi} l_{\tau}^{\eta}-\lambda \mu k_{\sigma}^{\alpha} l_{\tau}^{\beta}$.

THEOREM 1. The parameters $\lambda$ and $\mu$ being non-characteristic values of the kernels $K_{\sigma}^{\alpha}$ and $L_{r}^{\beta}$, respectively, a necessary and sufficient condition that (4) have a unique, continuous solution is that the parameter $\nu$ have a value which does not make null the Fredholm determinant of the kernel $N_{\sigma \tau}^{\alpha \beta}$ as given in (9). The solution is then given by (7).

It is to be noted that by taking the kernels of the transformations (5) in different order, that is for example

$z^{\alpha \beta}=y^{\alpha \beta}+N_{\sigma \tau}^{\alpha \beta} y^{\sigma \tau}, \quad \bar{z}^{\alpha \beta}=z^{\alpha \beta}+\lambda K_{\sigma}^{\alpha} z^{\sigma \beta}, \quad \bar{y}^{\alpha \beta}=\bar{z}^{\alpha \beta}+\mu L_{\tau}^{\beta} \bar{z}^{\alpha \tau}$,

we shall obtain the above theorem with a different permutation of functions, but in all instances the Fredholm determinant of the kernel $N_{\sigma \tau}^{\alpha \beta}$ so obtained reduces to exactly the same quantity.

COROLlaRY 1. The parameters $\lambda$ and $\mu$ being non-characteristic values of the kernels $K_{\sigma}^{\alpha}$ and $L_{r}^{\beta}$, respectively, a necessary and sufficient condition that the equation

$$
\bar{y}^{\alpha \beta}=y^{\alpha \beta}+\lambda K_{\sigma}^{\alpha} y^{\sigma \beta}+\mu L_{r}^{\beta} y^{\alpha \tau}
$$

have a unique, continuous inverse is that the Fredholm determinant of the kernel $-\lambda \mu k_{\sigma}^{\alpha} l_{\tau}^{\beta}$ be different from zero. In addition, for $\lambda$ and $\mu$ sufficiently small the solution will be given by

$$
\begin{aligned}
y^{\alpha \beta}=\bar{y}^{\alpha \beta}+\lambda k_{\sigma}^{\alpha} \bar{y}^{\sigma \beta}+\mu l_{\tau}^{\beta} \bar{y}^{a \tau}+\left\{2 R_{\sigma \tau}^{\alpha \beta}\left[-\lambda \mu k_{\rho}^{\xi} l_{\pi}^{\eta}\right]\right. \\
\\
\left.+\lambda k_{\xi}^{\alpha} R_{\sigma \tau}^{\xi \beta}+\mu l_{\eta}^{\beta} R_{\sigma \tau}^{\alpha \eta}\right\} \bar{y}^{\sigma \tau},
\end{aligned}
$$

where $R_{\sigma \tau}^{\alpha \beta}\left[-\lambda \mu k_{\rho}^{\xi} \eta_{\pi}^{\eta}\right]$ is the resolvent kernel of $-\lambda \mu k_{\sigma}^{\alpha} l_{\tau}^{\beta}$.

To prove the last part of the corollary, we note by hypothesis that*

$$
R_{\sigma \tau}^{\alpha \beta}\left[-\lambda \mu k_{\rho}^{\xi} l_{\pi}^{\eta}\right]=\lambda \mu k_{\sigma}^{\alpha} l_{\tau}^{\beta}+\lambda^{2} \mu^{2} k_{\xi}^{\alpha} k_{\sigma}^{\sigma} l_{\eta}^{\beta} \eta_{\tau}^{\eta}+\cdots,
$$

and so

$$
R_{\sigma \tau}^{\alpha \beta}=\lambda \mu k_{\xi}^{\alpha} \beta_{\eta}^{\beta} R_{\sigma \tau}^{\xi \eta}+\lambda \mu k_{\sigma}^{\alpha} l_{\tau}^{\beta} .
$$

\footnotetext{
* This is the usual series development of an inverse kernel.
} 
If the Fredholm determinant of the kernel $-\lambda \mu k_{\sigma}^{\alpha} l_{\tau}^{\beta}$ is zero, we see from (5) that the homogeneous equation (10), that is, $\bar{y}^{\alpha \beta} \equiv 0$, has a solution. Let us seek now to determine the nature of $\lambda$ and $\mu$ when this condition is fulfilled. For $\lambda$ and $\mu$ sufficiently small, the resolvent kernel of $-\lambda \mu k_{\sigma}^{\alpha} l_{\tau}^{\beta}$ is given by (11). To say that $D\left[-\lambda \mu k_{\sigma}^{\alpha} l_{\tau}^{\beta}\right]=0$ is to say that (11) or

$$
\bar{R}_{\sigma \tau}^{\alpha \beta} \equiv \frac{R_{o \tau}^{\alpha \beta}}{\lambda \mu}
$$

has a singularity for the particular values of $\lambda$ and $\mu$ taken.

By the Hadamard multiplication theorem, we know that the singular values of the series

$$
\begin{aligned}
\bar{R}_{\sigma \tau}^{\alpha \beta}(z)=k_{\sigma}^{\alpha} l_{\tau}^{\beta}+\lambda \mu k_{\xi_{1}}^{\alpha} k_{\sigma}^{\xi_{1}} l_{\eta_{1}}^{\beta} l_{\tau}^{\eta_{1}} z+\cdots \\
+\lambda^{n} \mu^{n} k_{\xi_{1}}^{\alpha} k_{\xi_{2}}^{\xi_{1}} \cdots k_{\sigma}^{\xi_{n}} l_{\eta_{1}}^{\beta} l_{\eta_{2}}^{\eta_{1}} \cdots l_{\tau}^{\eta_{n}} z^{n}+\cdots
\end{aligned}
$$

will be given by the product of the singularities of the two series

$$
k_{\sigma}^{\alpha}(z)=\sum_{i=0}^{\infty} \lambda^{i} k_{\xi_{1}}^{\alpha} k_{\xi_{2}}^{\xi_{1}} \cdots k_{\sigma}^{\xi_{i}} z^{i}, \quad l_{\tau}^{\beta}(z)=\sum_{i=0}^{\infty} \mu^{i} l_{\eta_{1}}^{\beta} l_{\eta_{2}}^{\eta_{1}} \cdots l_{\tau}^{\eta_{i}} z^{i} .
$$

By means of a well known relation satisfied by a resolvent kernel, namely

$$
\frac{d^{n}}{d \lambda^{n}}\left\{k_{\sigma}^{\alpha}\right\}=n ! k_{\xi_{1}}^{\alpha} k_{\xi_{1}}^{\xi_{1}} \cdots k^{\xi_{n}}
$$

we see that the equations (13) reduce to

(14) $k_{\sigma}^{\alpha}(z)=\sum_{i=0}^{\infty} \frac{\lambda^{i}}{i !} \frac{d^{i}}{d \lambda^{i}}\left\{k_{\sigma}^{\alpha}\right\} z^{i}, l_{\tau}^{\beta}(z)=\sum_{i=0}^{\infty} \frac{\mu^{i}}{i !} \frac{d^{i}}{d \mu^{i}}\left\{l_{\tau}^{\beta}\right\} z^{i}$.

Since the equations (14) are of the form of a MacLaurin's expansion, it is easily seen that the singular ensembles of $k_{\sigma}^{\alpha}(z)$ and $l_{\tau}^{\beta}(z)$ are given respectively by $z=\bar{\lambda} / \lambda-1$ and $z=\bar{\mu} / \mu-1$, where $\bar{\lambda}$ and $\bar{\mu}$ are characteristic values of the kernels $K_{\sigma}^{\alpha}$ and $L_{\tau}^{\boldsymbol{\beta}}$, respectively. Thus by Hadamard's theorem the singular ensemble of $(12)$ is given by $z=(\bar{\lambda} / \lambda-1)(\bar{\mu} / \mu-1)$. If we define $z=1$ to belong to this ensemble, then we see that $\lambda$ and $\mu$ must satisfy the relationship $\lambda / \bar{\lambda}+\mu / \bar{\mu}=1$. 
THEOREM 2. For $\lambda$ and $\mu$ sufficiently small and non-characteristic values of their respective kernels, a necessary and sufficient condition that the equation

$$
y^{\alpha \beta}+\lambda K_{\sigma}^{\alpha} y^{\sigma \beta}+\mu L_{\tau}^{\beta} y^{\alpha \tau}=0
$$

have a homogeneous solution is that the parameters satisfy a relation

$$
\frac{\lambda}{\bar{\lambda}}+\frac{\mu}{\bar{\mu}}=1,
$$

where $\bar{\lambda}$ and $\bar{\mu}$ are characteristic values of the kernels $K_{\sigma}^{\alpha}$ and $L_{\tau}^{\beta}$, respectively.

The sufficiency of the theorem is evident since (16) implies $\lambda \mu=(\bar{\lambda}-\lambda)(\bar{\mu}-\mu)$ and this in (12) is singular because of (14).

Let us now consider the ordinary associate equations with the kernels $K_{\sigma}^{\alpha}$ and $L_{\tau}^{\beta}$ respectively, that is,

$$
\left\{\begin{aligned}
\Psi_{\alpha}{ }^{i}+\bar{\lambda} K_{\alpha}{ }^{\sigma} \Psi_{\sigma}^{i} & =0, \quad(i=1,2, \cdots, n), \\
{ }^{\prime} \Psi_{\beta}{ }^{j}+\bar{\mu} L_{\beta}{ }^{\prime} \Psi_{\tau}{ }^{j} & =0, \quad(j=1,2, \cdots, m) .
\end{aligned}\right.
$$

These two equations have the solutions as indicated. In multiplying (15) separately by $\bar{\lambda} \Psi_{\alpha}^{i}$ and $\bar{\mu}^{\prime} \Psi_{\beta}{ }^{j}$ and integrating, we obtain the equations

$$
\begin{aligned}
\bar{\lambda} \Psi_{\alpha}^{i} y^{\alpha \beta}+\lambda \bar{\lambda} \Psi_{\alpha}^{i} K_{\sigma}^{\alpha} y^{\sigma \beta}+\bar{\lambda} \mu \Psi_{\alpha}^{i} L_{r}^{\beta} y^{\alpha \tau}=0, \\
\bar{\mu}^{\prime} \Psi_{\beta^{j}} y^{\alpha \beta}+\lambda \bar{\mu}^{\prime} \Psi_{\beta^{j}} K_{\sigma}^{\alpha} y^{\sigma \beta}+\mu \bar{\mu}^{\prime} \Psi_{\beta}^{j} L_{\tau}^{\beta} y^{\alpha \tau}=0 .
\end{aligned}
$$

These equations in virtue of (17) and (16) reduce to

$$
\Psi_{\alpha}^{i}\left\{y^{\alpha \beta}+\bar{\mu} L_{\tau}^{\beta} y^{\alpha \tau}\right\}=0, \quad{ }^{\prime} \Psi_{\beta}^{j}\left\{y^{\alpha \beta}+\bar{\lambda} K_{\sigma}^{\alpha} y^{\sigma \beta}\right\}=0 .
$$

It is evident that all solutions of (15) must satisfy simultaneously the equations (18); hence we have the following theorem.

Theorem 3. All solutions of (15), for a particular set of characteristic parameters $\bar{\lambda}$ and $\bar{\mu}$ satisfying (16), are of the form

$$
\sum_{i=1}^{n} \sum_{j=1}^{m} c_{i j} \Phi_{i}^{\alpha^{\prime}} \Phi_{j}{ }^{\beta}
$$

where the $c_{i j}$ are constants, and $\Phi_{i}{ }^{\alpha}$ and ${ }^{\prime} \Phi_{j}{ }^{\beta}$ are the respective fundamental solutions of the homogeneous equations

$$
\phi^{\alpha}+\bar{\lambda} K_{\sigma}^{\alpha} \phi^{\sigma}=0, \quad \phi^{\beta}+\bar{\mu} L_{\tau}^{\beta}{ }^{\prime} \phi^{\tau}=0 .
$$


With respect to the above theorem we must bear in mind that for an equation (15) with given parameter values $\lambda$ and $\mu$, there may exist more than one pair of characteristic parameters satisfying (16) and accordingly the totality of homogeneous solutions of (15) is enlarged.

It follows directly from the above theorem that the totality of solutions of the associate equation to (15), that is,

$$
y_{\alpha \beta}+\lambda K_{\alpha}^{\sigma} y_{\sigma \beta}+\mu L_{\beta}{ }^{\tau} y_{\alpha \tau}=0,
$$

have the form

$$
\sum_{i=1}^{n} \sum_{j=1}^{m} d_{i j} \Psi_{\alpha}^{i \prime} \Psi_{\beta}^{j}
$$

We have already noted the equivalence between (15) and the equation

$$
y^{\alpha \beta}-\lambda \mu k_{\sigma}^{\alpha} l_{r}^{\beta} y^{\sigma \tau}=0 .
$$

In like manner, we may show the equivalence between (19) and

$$
y_{\alpha \beta}-\lambda \mu k_{\alpha}{ }^{\sigma} l_{\beta}{ }^{\tau} y_{\sigma \tau}=0 .
$$

Since $\lambda$ and $\mu$ are not characteristic values of their respective kernels, by adding $\lambda \mu K_{\sigma}^{\alpha} L_{\tau}^{\beta} y^{\sigma \tau}$ to (10) and applying (3), we may write equation (10) in the form

$$
y^{\alpha \beta}-\lambda \mu k_{\sigma}^{\alpha} l_{\tau}^{\beta} y^{\sigma \tau}=\bar{y}_{\sigma}^{\alpha \beta}+\lambda k_{\sigma}^{\alpha} \bar{y}^{\sigma \beta}+\mu l_{\tau}^{\beta} \bar{y}^{\alpha \tau}+\lambda \mu k_{\sigma}^{\alpha} l_{\tau}^{\beta} \bar{y}^{\sigma \tau} .
$$

A necessary and sufficient condition that (22) have a solution when $\lambda$ and $\mu$ satisfy (16) is that all solutions of (21) when composed with the right hand side of (22) yield zero. Furthermore, from the identities

$$
\Psi_{\sigma}^{i} k_{\alpha}{ }^{\sigma}=\frac{1}{\bar{\lambda}-\lambda} \Psi_{\alpha}^{i}, \quad{ }^{\prime} \Psi_{\sigma}^{j} l_{\alpha}{ }^{\sigma}=\frac{1}{\bar{\mu}-\mu}{ }^{\prime} \Psi_{\alpha}^{j},
$$

we see that a necessary and sufficient condition that the equation (10) have a solution when the parameters satisfy a relationship (16) is that the function $\bar{y}^{\alpha \beta}$ satisfy the $n \cdot m$ equations

$$
\Psi_{\alpha}^{i \prime} \Psi_{\beta}^{j} \bar{y}^{\alpha \beta}=0, \quad(i=1,2, \cdots, n ; j=1,2, \cdots, m) .
$$

The actual solutions may be computed from the equation (22) in the usual fashion. 
Lemma. A necessary and sufficient condition that

$$
A^{\alpha \beta} y^{\alpha \beta}+B_{\sigma}^{\alpha \beta} y^{\sigma \beta}+C_{\tau}^{\alpha \beta} y^{\alpha \tau}+D_{\sigma \tau}^{\alpha \beta} y^{\sigma \tau}=0
$$

be true for all continuous functions $y^{\alpha \beta}$ is that

$$
A^{\alpha \beta} \equiv 0, B_{\sigma}^{\alpha \beta} \equiv 0, C_{\tau}^{\alpha \beta} \equiv 0, D_{\tau \sigma}^{\alpha \beta} \equiv 0
$$

in the field of continuous functions.

This lemma may be easily verified by taking $y^{\alpha \beta}=\phi^{\alpha} \psi^{\beta}$ and applying repeatedly a known lemma* that if

$$
P^{\alpha} f^{\alpha}+Q_{\sigma}^{\beta} f^{\sigma}=0
$$

be true for all continuous functions $f^{\alpha}$, then $P^{\alpha} \equiv 0, Q_{\sigma}^{\alpha} \equiv 0$.

In virtue of the transitivity of equations of the form (4) and the preceding lemma, we see that a necessary and sufficient condition that

$$
y^{\alpha \beta}=\bar{y}^{\alpha \beta}+\lambda k_{\sigma}^{\alpha} \bar{y}^{\sigma \beta}+\mu l_{\tau}^{\beta} \bar{y}^{\alpha \tau}+m_{\tau \sigma}^{\alpha \beta} \bar{y}^{\sigma \tau},
$$

where we have assumed $\lambda$ and $\mu$ to have non-characteristic values, be an inverse to (4) is that the kernel $M_{\sigma \tau}^{\alpha \beta}$ satisfy the relations

$$
\left\{\begin{array}{c}
m_{\sigma \tau}^{\alpha \beta}+\nu m_{\sigma \tau}^{\alpha \beta}+\nu m_{\xi \eta}^{\alpha \beta} M_{\sigma \tau}^{\xi \eta}+\lambda \nu k_{\xi}^{\alpha} M_{\sigma \tau}^{\xi \beta}+\mu \nu l_{\eta}^{\beta} M_{\sigma \tau}^{\alpha \eta}+\lambda m_{\xi \tau}^{\alpha \beta} K_{\sigma}^{\xi} \\
\quad+\mu m_{\sigma \eta}^{\alpha \beta} L_{\tau}^{\eta}+\lambda \mu k_{\sigma}^{\alpha} L_{\tau}^{\beta}+\lambda \mu K_{\sigma}^{\alpha} l_{\tau}^{\beta}=0 \\
m_{\sigma \tau}^{\alpha \beta}+\nu m_{\sigma \tau}^{\alpha \beta}+\nu m_{\xi \eta}^{\alpha \beta} M_{\sigma \tau}^{\xi \eta}+\lambda \nu M_{\xi \tau}^{\alpha \beta} k_{\sigma}^{\xi}+\mu \nu M_{\sigma \eta}^{\alpha \beta} l_{\tau}^{\eta}+\lambda K_{\xi}^{\alpha} m_{\sigma \tau}^{\xi \beta} \\
\quad+\mu L_{\eta}^{\beta} m_{\sigma \tau}^{\alpha \eta}+\lambda \mu k_{\sigma}^{\alpha} L_{\tau}^{\beta}+\lambda \mu K_{\sigma}^{\alpha} l_{\tau}^{\beta}=0
\end{array}\right.
$$

THEOREM 4. The parameters $\lambda$ and $\mu$ being non-characteristic and satisfying no relationship (16), a necessary and sufficient condition that a linear integral equation of the form

$$
\bar{y}^{\alpha \beta}=y^{\alpha \beta}+\lambda K_{\sigma}^{\alpha} y^{\sigma \beta}+\mu L_{\tau}^{\beta} y^{\alpha \tau}
$$

have a solution in the same form is that the kernels $K_{\sigma}^{\alpha}$ and $L_{\tau}^{\beta}$ satisfy conditions

(24) $c K_{\sigma}^{\alpha}+K_{\sigma}^{\alpha}+c \lambda K_{\xi}^{\alpha} K_{\sigma}^{\xi}=0, \quad c L_{\tau}^{\beta}-L_{\tau}^{\beta}+c \mu L_{\eta}^{\beta} L_{\tau}^{\eta}=0$,

* See Michal and Peterson, The invariantive theory of functional forms under the group of linear functional transformations of the third kind, Annals of Mathematics, vol. 32 (1932), p. 432. 
simultaneously, where $c$ is a constant.

From (23) we see by hypothesis that we must have

$$
k_{\sigma}^{\alpha} L_{\tau}^{\beta}+K_{\sigma}^{\alpha} l_{\tau}^{\beta}=0 .
$$

Dividing this equation by $K_{\sigma}^{\alpha} L_{\tau}^{\beta}$, we see that it is necessary that

$$
k_{\sigma}^{\alpha}=c K_{\sigma}^{\alpha}, \quad l_{\tau}^{\beta}=-c L_{\tau}^{\beta},
$$

where $c$ is a constant. Substituting these values of the resolvent kernels in the well known relation satisfied by a kernel and its resolvent, we obtain (24). The sufficiency of the theorem is easily shown by noting that the hypotheses of the theorem imply from (23) that

$$
m_{\sigma \tau}^{\alpha \beta}+\lambda K_{\xi}^{\alpha} m_{\sigma \tau}^{\xi \beta}+\mu L_{\eta}^{\beta} m_{\sigma \tau}^{\alpha \eta}=0 .
$$

Since $\lambda$ and $\mu$ satisfy no relation of the form (16), by Corollary 1 , it follows that $m_{\sigma \tau}^{\alpha \beta} \equiv 0$.

As an example of kernels whose resolvent kernel is just a constant times the kernel itself, consider the kernel $K_{\sigma}^{\alpha}=A^{\alpha} B_{\sigma}$; then $k_{\sigma}^{\alpha}=-K_{\sigma}^{\alpha} /\left(1+K_{\xi}^{\xi}\right)$. If the kernels $K_{\sigma}^{\alpha}$ and $L_{r}^{\beta}$ of the above theorem are of this type, then it is necessary that

$$
K_{\sigma}{ }^{\sigma}+L_{\sigma}{ }^{\sigma}=-2,
$$

in order that the theorem apply.

In concluding this paper, it is of interest to note that the linear integral equation

$$
\bar{y}^{\alpha \beta}=A^{\alpha \beta} y^{\alpha \beta}+\lambda B_{\sigma}^{\alpha \beta} y^{\sigma \beta}+\mu C^{\alpha \beta} y^{\alpha \tau}+\nu D_{\tau \sigma}^{\alpha \beta} y^{\sigma \tau}
$$

may be reduced to the form (4) provided the kernels of (25) are of such a nature that $B_{\sigma}^{\alpha \beta} / A^{\alpha \beta}$ is free of $\beta, C_{r}^{\alpha \beta} / A^{\alpha \beta}$ is free of $\alpha$, and $A^{\alpha \beta} \neq 0,(a \leqq \alpha, \beta \leqq b)$.

Los Angeles, California 